\title{
Editorial: Mobile Networks and Management
}

\author{
Ramón Agüero $^{1} \cdot$ Thomas Zinner $^{2} \cdot$ Mario Garcia-Lozano ${ }^{3} \cdot$ Bernd-Ludwig Wenning $^{4}$. \\ Andreas Timm-Giel ${ }^{5}$
}

Published online: 6 July 2016

(C) Springer Science+Business Media New York 2016

\section{Guest editorial}

Since its initial edition in 2009, the Mobile Networks and Management (MONAMI) conference has aimed at providing a meeting point for researchers and people working on the mobile and wireless networking realm. The rapid advances of the corresponding technologies together with the increasing demands from the end users and the appearance of new services, are some of the causes of the ever evolving requirements and challenges that need to be tackled. Together with the conference, we started with a series of Special Issues (SI) of the Mobile Networks and Applications Journal in 2010. This series gives an overview of some of the most interesting currently active research challenges and issues. The call-for-papers of this SI was an outcome of the 7th edition of the MONAMI conference, which was held at the University of Cantabria, Spain, in September 2015. Thirty-three papers were received and reviewed by at least three independent and reputable referees. As a result of the process, six manuscripts were selected to be included in this SI. We believe that they provide a very good picture on the current situation of research within some of the various

Ramón Agüero

ramon@tlmat.unican.es

\footnotetext{
University of Cantabria, Santander, Spain

University of Würzburg, Würzburg, Germany

Technical University of Catalunya, Barcelona, Spain

4 Cork Institute of Technology, Cork, Ireland

5 Hamburg University of Technology, Hamburg, Germany
}

topics within the broad field of wireless networks and their management.

In the first paper of the SI, entitled Introducing Mobile Edge Computing Capabilities through Distributed $5 G$ Cloud Enabled Small Cells, Jose-Oscar Fajardo et al. address one of the key elements of the forthcoming 5G systems: the virtualization of network functions from the base stations to the cloud. It goes without saying that this is attracting a lot of attention, not only from the scientific community, but also from the standardisation bodies, telecom operators and vendors. In this work, the authors exploit this shifting of functions and storage capabilities to the mobile edge in order to optimize services running on top of the network. The paper discusses that a centralized approach is not always feasible, due to the limited capacities of the fronthaul connections, being especially relevant for small cell deployments, leading to the so-called cloud-enabled Small Cells. In order to address these particular cases, the authors propose placing processing and storage capabilities closer to the remote units. An architecture to support such functionality is presented and relevant application scenarios are discussed, including the support of multi-tenancy. The paper discusses these ideas that are being considered in the framework of the H2020 5GPP project "Small cEllS coordinAtion for Multi-tenancy and Edge services" (SESAME).

The increase in the number of users, devices, and network elements has led to a remarkable increase of energy expenditure and, consequently, the operational expenses. As a consequence, the scientific community has put, in recent years, considerable effort into the promotion of energy-aware communications and networking. In the second paper of this SI, E. Benkhelifa et al. carry out a thorough review of how different works have addressed the modelling of power consumption for mobile devices, to propose solutions aimed at an energyaware network operation. The extensive survey carried out 
allows the authors to identify some of the existing gaps, which are a consequence of novel solutions, such as Distributed Systems, Mobile Cloud Computing, Internet of the Things (IoT) and Software Defined Networking (SDN). Based on these findings, the authors claim that a unified view on energy modelling for network simulations is still required, proposing an initial number of considerations that should be taken into account.

In order to be able to address the growing capacity demand, forthcoming cellular systems will need to foster novel solutions, including the densification of the access networks with small cells as well as the possibility to enable direct communications between end-user devices (Device-To-Device, D2D). Although it is believed that these approaches are mandatory to face the stringent capacity needs, they will also bring new challenges. In the third paper of this special issue, entitled Joint User Association and Interference Mitigation for D2DEnabled Heterogeneous Cellular Networks, T. Zhou et al. present a downlink load balancing scheme to be used over a scenario comprising both small cells and D2D communications, jointly considering user-cell association and D2D mode selection. In addition, they introduce a resource partitioning scheme to mitigate the interference suffered from the users that are off-loaded from the high-power base stations. An optimization problem is posed, and the paper presents an algorithm that could be executed in a distributed way, way allowing a scalable and fast optimization of the resources. A system-level evaluation is used to assess the performance of the proposed scheme.

The paper presented in the above paragraph assumes that both the small cells and the D2D communications use the same technology, and resources, as the available base stations. Another approach that is currently fostered by mobile operators is to offload traffic to other technologies, in particular WiFi. Hence, the use of IEEE 802.11 networks in outdoor scenarios is gaining relevance. In the fourth paper of this Special Issue, D. Bri et al. show a study carried out over a real testbed to analyse the impact of weather conditions over the performance of IEEE 802.11 communications. Using a statistical approach, they group weather conditions, studying how they affect the performance of the communications. They carry out a very extensive measurement campaign exploiting an SNMP-based monitoring solution. In this sense, the paper goes beyond previous works, which are mostly based on either simulations or time-limited experiments. Unexpectedly, the results show that shorter links are more severely jeopardized by adverse conditions, due to the modulation schemes that are used over such short links.
The last two papers on this special issue focus on MachineTo-Machine (M2M) communications and IoT. Their presence is expected to outstandingly grow in the forthcoming years and, due to their particular characteristics, they might require some networking solutions that are not suitable for other types of systems. First, F-H. Kumbhar et al. introduce, in the fifth paper, RoBiN, a Random Access Scheme Based on Border Routers with Complete Frequency Reuse Capability. The authors argue that the expected large number of devices that will need to access the network due to the massive deployment of M2M could cause a relevant degradation of the performance of the random access channel. They exploit the so-called border routers, which are similar to small cells. By means of both a mathematical model and a simulation campaign using the ns-3 framework, the paper shows that the proposed scheme outperforms legacy systems both in terms of collision probability and system performance.

In the last paper of this SI, Optimised Joint Channel Network Coding for Sensor Network with Correlated Sources, S-Z. Ammar et al. discuss a physical layer network coding scheme to be used over wireless sensor networks where sources are assumed to be correlated. These are supposed to send the information to a single receiver by means of a relaying station. The authors evaluate their proposed scheme, comparing it with traditional solutions, in which the correlation between sources is not exploited. The presented solution clearly outperforms these alternatives, both over AWGN and Rayleigh channels.

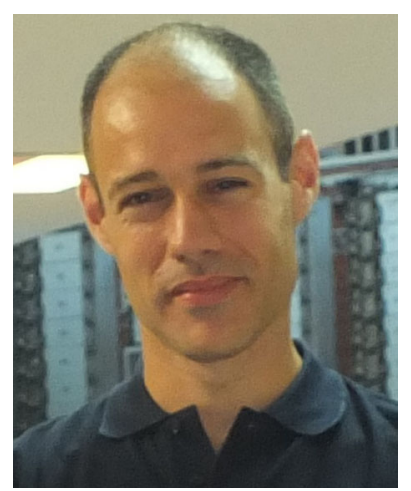

Ramón Agüero received a degree in Telecommunications Engineering from the University of Cantabria in 2001 and the $\mathrm{PhD}$ in 2008. He is currently an Associate Professor at the Communications Engineering Department at that university. Since 2016 he is also the Head of the Campus Area. He has participated in several collaborative research projects and his research focuses on future network architectures, especially regarding the (wireless) access part of the network. He is also interested on multi-hop (mesh) networks, device-todevice communications, and Network Coding. He has published more than 160 technical papers in such areas and he is a regular TPC member and reviewer on various related conferences and journals. 


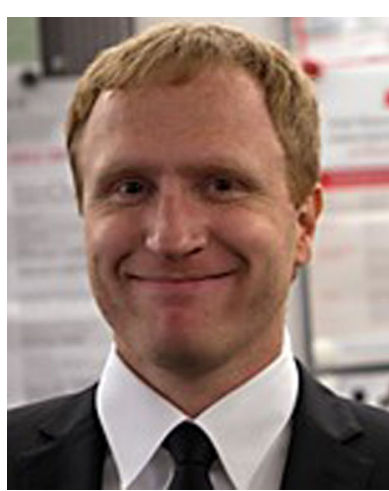

Thomas Zinner is a postdoctoral researcher at the chair of Communication Networks at the University of Würzburg. He received his diploma degree in Computer Science from the University of Würzburg in 2006 and his $\mathrm{PhD}$ in 2012. Since then he heading the research group on "Next Generation Networks" at the chair of Communication Networks at the University of Würzburg. He is acting as MC member and Working Group Chair in the COST action IC1303 AAPELE. His research interests cover performance evaluation and modelling of future internet architectures with emphasis on SoftwareDefined Networking and Network Function Virtualization. He is also interested in video streaming techniques, Quality-of-Experience, and application-network interaction.

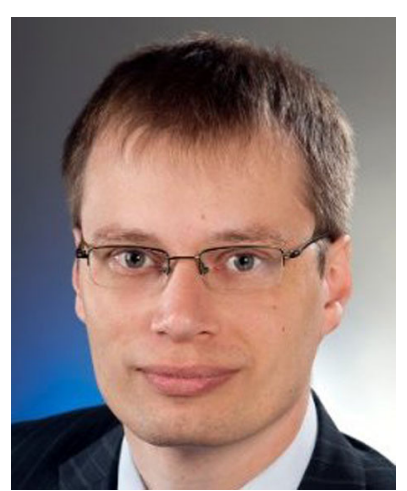

Bernd-Ludwig Wenning received Dipl.-Ing. and Dr.-Ing. degrees in Electrical Engineering and Information Technology from University of Bremen, Germany, in 2002 and 2009. From 2002 to 2012, he was a researcher at University of Bremen, participating in several projects in the area of communication networks. In 2012, he joined Nimbus Centre at Cork Institute of Technology, Ireland, as a Research Fellow. Since then, he has been involved in a number of national and European projects around wireless sensor networks. Throughout his research career, Bernd-Ludwig Wenning has published more than 40 papers in conferences and journals.

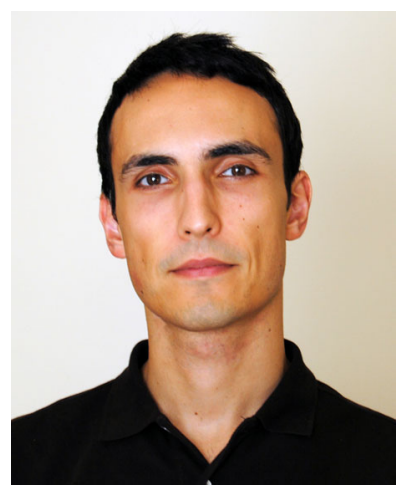

Mario Garcia-Lozano received his M.Sc. and Ph.D. in Telecommunications Engineering from the Universitat Politecnica de Catalunya (UPC, BarcelonaTECH), Spain, in 2001 and 2009 respectively. From 1999 to 2002 he was a member of the technical staff at Retevision, Spain where he worked on the radio planning of LMDS networks. In 2002 he joined the Department of Signal Theory and Communications at UPC, where he currently lectures as a tenured associate professor. He has actively participated in several research projects funded by the European Union and the Spanish government. He has also worked as a consultant in projects funded by private telecommunication companies and institutions. His research activities are focused in the field of radio network planning and radio resource management issues both for cellular and broadcasting networks.

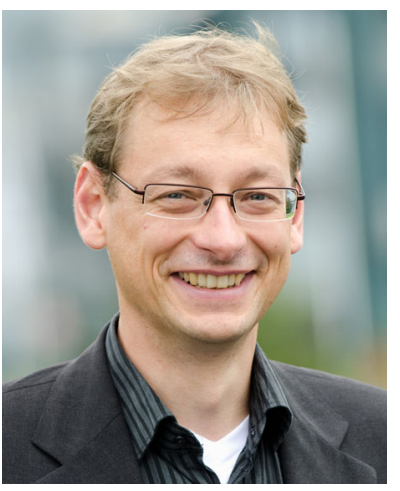

Andreas Timm-Giel was, between 1994 and 1999, group leader at the University of Bremen in the area of mobile and satellite communications and involved in several EU funded projects. After receiving his $\mathrm{PhD}$ in 1999 he moved to MediaMobil $\mathrm{GmbH}$ and M2SAT Ltd. as Technical Project Leader and Manager Network Operations. In December 2002 he joined the Communication Networks group at the University of Bremen as senior researcher and lecturer. $\mathrm{He}$ was leading several industrial, national and EC funded research projects and from 2006 he was additionally directing the interdisciplinary activity "Adaptive Communications" of TZI (Center of Computing and Communication Technologies). In November 2009 he was appointed full professor at Hamburg University of Technology (TUHH) and is heading the Institute of Communication Networks. Since 2012 he is coordinator of the research cluster SOMSED "Self-organizing mobile sensor and data networks" at TUHH and from 2013 to 2014 deputy head of the TUHH's school of electrical engineering, computer science and mathematics. Since October 2014 he is Vice President for Research at TUHH. His research interests are mobile and wireless communications, sensor networks and the Future Internet. 